\title{
Left Ventricle Non-Compaction Cardiomyopathy Admitted With Multiorgan Failure: A Case Report
}

\author{
Luis F. Álvarez Pérez ${ }^{1}$, Jorge E. Sandelis Pérez ${ }^{1}$, Juan Nieves-Rivera ${ }^{2}$, Hilton Franqui ${ }^{2}$ \\ 1. Internal Medicine, University of Puerto Rico, Medical Sciences Campus, San Juan, PRI 2. Cardiology, University of \\ Puerto Rico, Medical Sciences Campus, San Juan, PRI
}

Corresponding author: Luis F. Álvarez Pérez, luis.alvarez22@upr.edu

\begin{abstract}
Left ventricle non-compaction (LVNC) is a rare congenital cardiomyopathy characterized by thickened myocardium due to an arrest of the normal compaction of the embryonic sponge-like meshwork of myocardial fibers.

We present a 40-year-old man with no known systemic illnesses admitted with cardiogenic shock and multiorgan failure. Echocardiogram revealed severe enlargement of all four chambers with left ventricular ejection fraction (LVEF) $<10 \%$. Cardiac magnetic resonance imaging (CMR) showed hypertrabecular left ventricular myocardium with a ratio of non-compact to compact myocardium of 2.3, diffuse myocardial thinning, and a 16-mm left ventricular thrombus. These findings were compatible with LVNC. The patient was treated with intravenous inotropic vasopressors for cardiogenic shock and enoxaparin as bridging for warfarin to a goal of INR 2.0-3.0. Due to refractory heart failure (HF) and dependency on inotropic support, the patient was placed on the waiting list for a heart transplant. Unfortunately, 27 days after admission, he presented ventricular tachycardia arrest and did not respond to aggressive advanced cardiac life support measures.
\end{abstract}

A high index of suspicion is required for the early diagnosis, which in turn allows the physician to prevent complications of this condition. There is no specific therapy, so management is directed toward the clinical manifestations including HF, arrhythmias, and systemic embolic events. Heart transplantation is the only definitive treatment.

Received 05/08/2020

Review began 05/24/2020

Review ended 06/09/2020

Published 06/23/2020

๑) Copyright 2020

Álvarez Pérez et al. This is an open access article distributed under the terms of the Creative Commons Attribution License CC-BY 4.0., which permits unrestricted use, distribution, and reproduction in any medium, provided the original author and source are credited.
Categories: Cardiac/Thoracic/Vascular Surgery, Cardiology, Internal Medicine

Keywords: congenital, cardiomyopathy, left ventricle, non-compaction, heart failure, ventricular tachycardia

\section{Introduction}

Left ventricle non-compaction (LVNC) is a very rare congenital cardiomyopathy characterized by thickened myocardium due to an arrest of the normal compaction of the embryonic sponge-like meshwork of myocardial fibers [1-4]. The first isolated case was described in 1984 by Engberding, and its prevalence among patients undergoing echocardiography is estimated at $0.014-1.3 \%$ [5-7] and 3-4\% in patients with heart failure (HF) [8].

A high index of suspicion is required for the early diagnosis, which in turn allows the physician to prevent complications of this condition such as fatal arrhythmias, thromboembolic phenomenon, severe HF, and sudden cardiac death.

We present a complex case admitted with cardiogenic shock in the setting of de novo HF secondary to LVNC.

\section{Case Presentation}

A 40-year-old man with no known systemic illnesses presented with a four-week history of progressive shortness of breath and lower extremity swelling. Associated symptoms included dyspnea on minimal exertion, orthopnea, and paroxysmal nocturnal dyspnea. Vital signs were remarkable for a blood pressure of $90 / 40 \mathrm{mmHg}$, tachycardia of $110 \mathrm{bpm}$, and pulse oximetry of $92 \%$. Physical examination was relevant for anasarca, venous jugular pressure of $12 \mathrm{mmHg}$, diffuse bilateral inspiratory wet pulmonary crackles, a third heart sound (S3), a grade II/VI systolic murmur at the apex, and cold extremities. Laboratory tests were consistent with acute kidney injury (serum creatinine of $1.7 \mathrm{mg} / \mathrm{dL}$ and urea nitrogen of $49 \mathrm{mg} / \mathrm{dl}$ ) and markedly elevated liver enzymes with aspartate aminotransferase of $5366 \mathrm{IU} / \mathrm{L}$ and alanine aminotransferase of $4967 \mathrm{IU} / \mathrm{L}$. These findings were consistent with multiorgan failure. A chest X-ray revealed pulmonary vascular congestion (Figure 1). 


\section{Cureus}

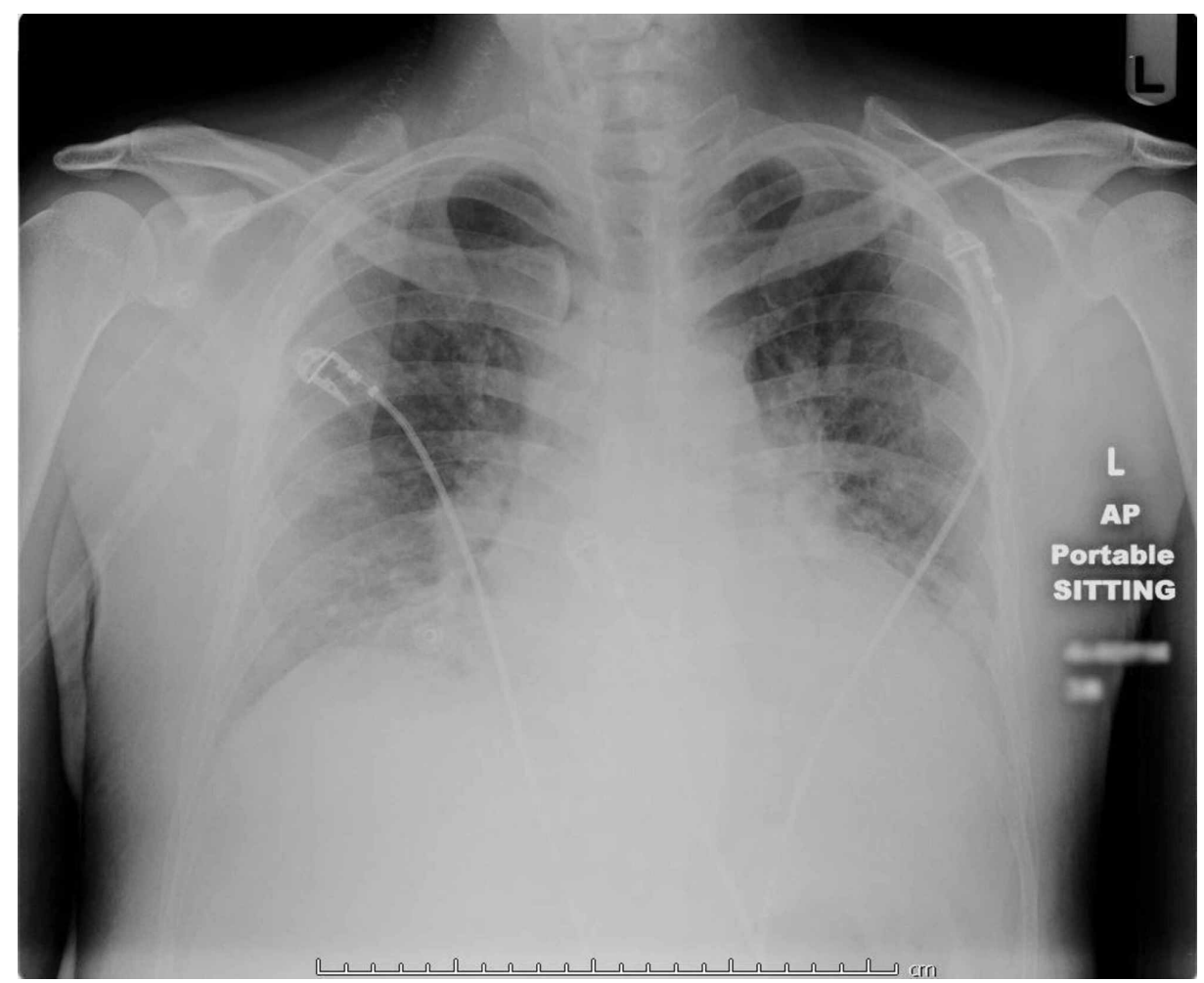

\section{FIGURE 1: Portable CXR (single view)}

The X-ray shows cardiomegaly and mild pulmonary vascular congestion centrally, indicating early CHF changes; additionally, there are no consolidations, no pneumothorax, and the anterior pleura spaces are clear. The bones are grossly intact.

CXR: chest X-ray; CHF: congestive heart failure

The echocardiogram showed severe mitral regurgitation and tricuspid regurgitation, with severe enlargement of all four chambers, and left ventricular ejection fraction (LVEF) $<10 \%$ (Figure 2). The patient was admitted to the cardiac care unit. 


\section{Cureus}
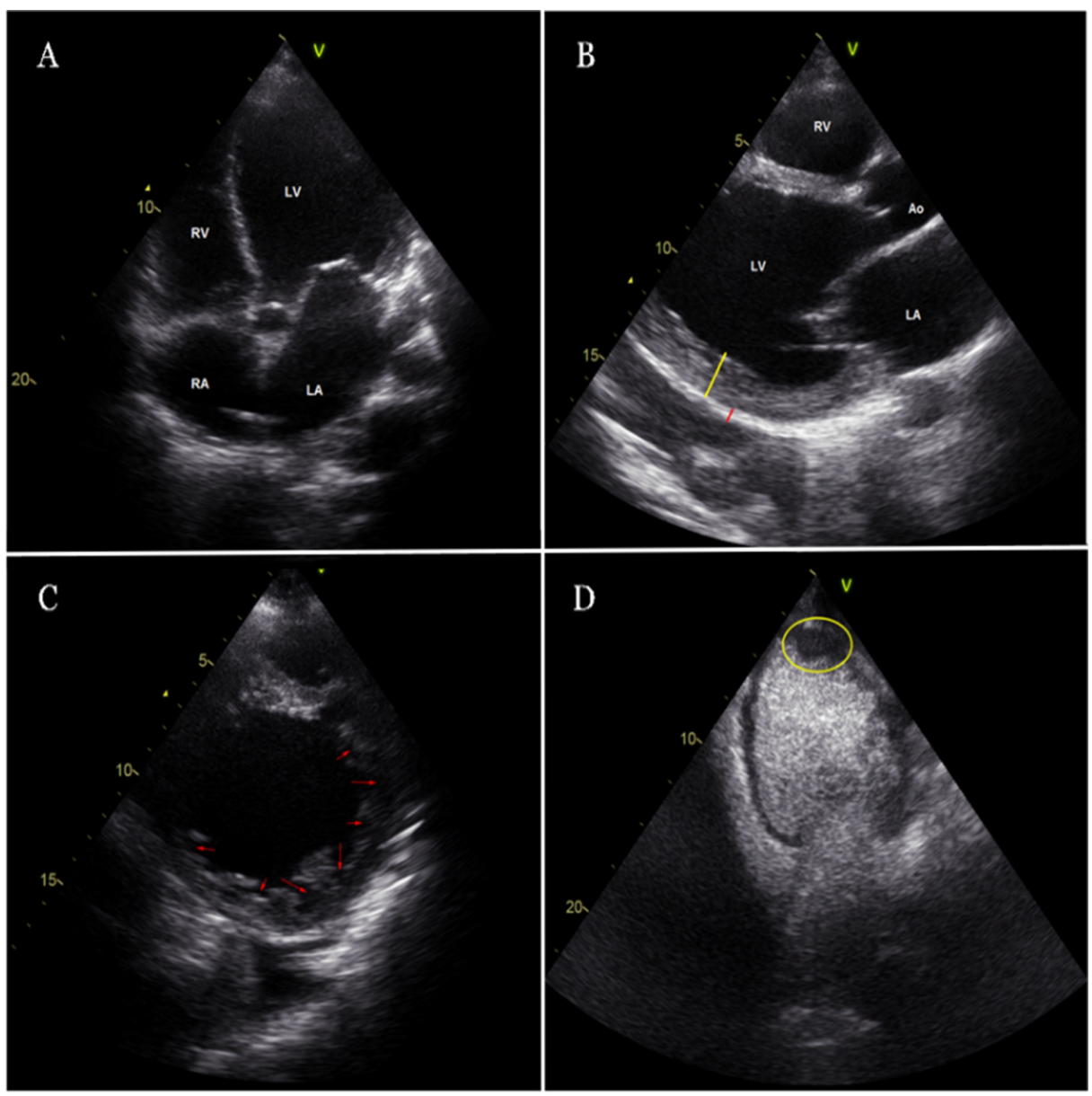

\section{FIGURE 2: 2D echocardiogram}

Apical four-chamber view (A) showing severe enlargement of all four chambers, a thin compacted epicardial layer (red line) and a markedly thickened endocardial layer (yellow line) with a maximum ratio of noncompacted to compacted myocardium >2:1 can be appreciated on the parasternal long-axis view (B); the parasternal short-axis view (C) is pertinent for numerous prominent trabeculations and deep recesses (red arrows). On apical two-chamber view (D) there is an apical left ventricular thrombus (yellow circle).

Cardiac catheterization demonstrated normal coronary arteries and reversible mild pulmonary hypertension.

Cardiac magnetic resonance imaging (CMR) (Figure 3) showed depressed biventricular systolic function (LVEF < $10 \%$; RVEF < $15 \%$ ), hypertrabecular left ventricular myocardium with a ratio of non-compact to compact myocardium of 2.3, diffuse myocardial thinning, and a 16-mm left ventricular thrombus. These findings were compatible with LVNC. As mentioned above, the patient had no history of heart disease, and no precipitating factor for his new-onset HF was identified during admission process.
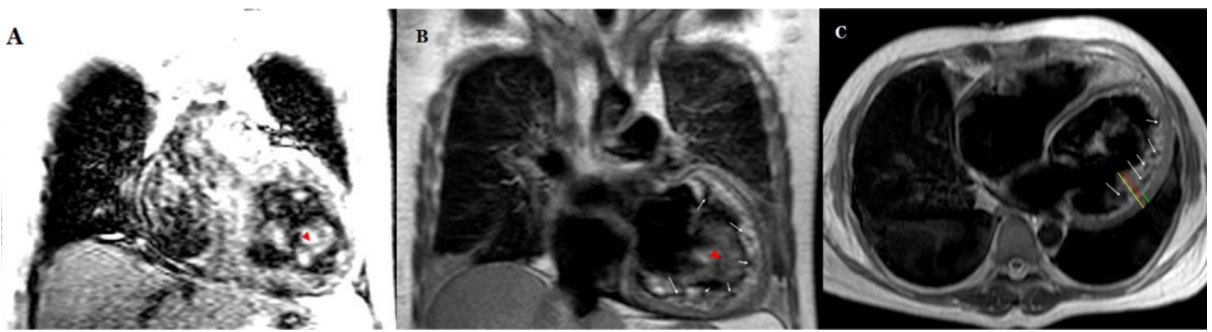

\section{FIGURE 3: Cardiac magnetic resonance imaging}

(A) and (B) (coronal Views) showed left ventricular intracavitary thrombus identified in the apical lateral wall, measuring $16 \mathrm{~mm}$ (red arrowhead), with hypertrabeculation and deep myocardial intratrabecular recesses; (B) and (C) (white arrows), the ratio of the compacted to non-compacted myocardium was 2.3 (C) (Axial view) (lines yellow, red, and green) 
Extremity venous Doppler revealed bilateral lower extremity posterior tibial and peroneal deep venous thrombosis, as well as upper extremity cephalic and basilic vein thrombosis.

The patient was treated with intravenous inotropic vasopressors for cardiogenic shock and enoxaparin as bridging for warfarin to a goal of INR 2.0-3.0. The patient was placed on the waiting list for heart transplant due to refractory HF and dependency on inotropic support. Unfortunately, 27 days after admission, he presented with ventricular tachycardia arrest and did not respond to aggressive advanced cardiac life support measures.

\section{Discussion}

LVNC is thought to be caused by an arrest of the normal compaction of the embryonic sponge-like meshwork of interwoven myocardial fibers during embryogenic life, resulting in thickened myocardium with two layers consisting of non-compacted myocardium and a thin compacted layer of myocardium [9]. The presence of deep endomyocardial intertrabecular recesses, which are filled with blood from the ventricular cavity, is typical of this condition and predisposes to embolic events [1-4].

This condition may sporadically develop in individuals without family history, although an autosomal dominant is the most commonly observed pattern of inheritance [10]. More than 10 genetic mutations have been identified, especially in genes encoding for mitochondrial, Z-line, sarcomeric, and cytoskeletal proteins [11].

Its clinical manifestations are highly variable, ranging from no symptoms to disabling congestive HF, lifethreatening ventricular arrhythmias, and systemic thromboembolism [5,12]. However, there is no gold standard for diagnosis of LVNC; the diagnosis should be suspected based on morphologic criteria for noncompaction or hypertrabeculation in patients with or without cardiac symptoms [13].

Echocardiogram is the most used imaging modality both to establish the diagnosis and for the follow-up $[5,14,15]$. The most widely accepted echocardiographic criteria are the Jenni Criteria (Table.1) and all four are required for diagnosis [15].

\section{Jenni Criteria for LVNC}

A thickened LV wall consisting of two layers: a thin compacted epicardial layer and a markedly thickened endocardial layer with numerous prominent trabeculations and deep recesses with a maximum ratio of non-compacted to compacted myocardium $>2: 1$ at end-systole in the parasternal short-axis view.

Color Doppler evidence of flow within the deep intertrabecular recesses.

Prominent trabecular meshwork in the LV apex or midventricular segments of the inferior and lateral wall.

Compacted wall thickness $\leq 8.1 \mathrm{~mm}$. The criterion of maximal systolic compacta thickness of $\leq 8.1 \mathrm{~mm}$ was found to be very specific for myocardial thickening in LVNC compared to normal controls or patients with aortic stenosis.

\section{TABLE 1: Echocardiographic criteria for diagnosis of LVNC}

Adapted from Jenni et al. [15].

LV: left ventricular; LVNC: left ventricle non-compaction.

When echocardiographic findings are not definitive, CMR criteria can be used to support the diagnosis of LVNC; among them, the maximum end-diastolic non-compacted to compacted myocardial thickness ratio of $>2.3$ and a trabeculated LV mass $>20 \%$ of global LV mass $[16,17]$. However, the sensitivity and specificity are complex to determine, and due to the risk of overestimation, these criteria should be used with caution.

There is no specific therapy, so management is directed toward the clinical manifestations including HF, arrhythmias, and systemic embolic events. Heart transplantation is the only definitive treatment. Attending to the risk of cardiac sudden death, implantable cardioverter-defibrillator may have a role in these cases [18].

\section{Conclusions}

Because of its low prevalence, the diagnosis of LVNC cardiomyopathy is often missed or delayed in adults. A high index of suspicion is required for the early diagnosis, and prompt referral to the cardiologist in order to ensure the most appropriate management and follow-up of these patients. 


\section{Additional Information \\ Disclosures}

Human subjects: Consent was obtained by all participants in this study. Conflicts of interest: In compliance with the ICMJE uniform disclosure form, all authors declare the following: Payment/services info: All authors have declared that no financial support was received from any organization for the submitted work. Financial relationships: All authors have declared that they have no financial relationships at present or within the previous three years with any organizations that might have an interest in the submitted work. Other relationships: All authors have declared that there are no other relationships or activities that could appear to have influenced the submitted work.

\section{References}

1. Ritter M, Oechslin E, Sütsch G, Attenhofer C, Schneider J, Jenni R: Isolated noncompaction of the myocardium in adults. Mayo Clin Proc. 1997, 72:26-31. 10.4065/72.1.26

2. Weiford BC, Subbarao VD, Mulhern KM: Noncompaction of the ventricular myocardium. Circulation. 2004, 109:2965-2971. 10.1161/01.CIR.0000132478.60674.D0

3. Arbustini E, Weidemann F, Hall JL: Left ventricular noncompaction: a distinct cardiomyopathy or a trait shared by different cardiac diseases?. J Am Coll Cardiol. 2014, 64:1840-1850. 10.1016/j.jacc.2014.08.030

4. Peritz DC, Vaughn A, Ciocca M, Chung EH: Hypertrabeculation vs left ventricular noncompaction on echocardiogram: a reason to restrict athletic participation?. JAMA Intern Med. 2014, 174:1379-1382. 10.1001/jamainternmed.2014.1066

5. Goud A, Padmanabhan S: A rare form of cardiomyopathy: left ventricular non-compaction cardiomyopathy . J Community Hospital Internal Med Persp. 2016, 6:1-5. 10.3402/jchimp.v6.29888

6. Aras D, Tufekcioglu O, Ergun K, et al.: Clinical features of isolated ventricular noncompaction in adults long-term clinical course, echocardiographic properties, and predictors of left ventricular failure. J Card Fail. 2006, 12:726-733. 10.1016/j.cardfail.2006.08.002

7. Stanton C, Bruce C, Connolly H, et al.: Isolated left ventricular noncompaction syndrome . Am J Cardiol. 2009, 104:1135-1138. 10.1016/j.amjcard.2009.05.062

8. Kovacevic-Preradovic T, Jenni R, Oechslin EN, Noll G, Seifert B, Attenhofer CH: Isolated left ventricular noncompaction as a cause for heart failure and heart transplantation: a single center experience. Cardiology. 2009, 112:158-164. 10.1159/000147899

9. Chin TK, Perloff JK, Williams RG, Jue K, Mohrmann R: Isolated noncompaction of left ventricular myocardium. A study of eight cases. Circulation. 1990, 82:507-513. 10.1161/01.cir.82.2.507

10. Zaragoza MV, Arbustini E, Narula J: Noncompaction of the left ventricle: primary cardiomyopathy with an elusive genetic etiology. Curr Opin Pediatrics. 2007, 19:619-627. 10.1097/MOP.0b013e3282f1ecbc

11. Richard P, Ader F, Roux M, et al.: Targeted panel sequencing in adult patients with left ventricular noncompaction reveals a large genetic heterogeneity. Clin Genet. 2019, 95:356-367. 10.1111/cge.13484

12. Engberding R, Bender F: Identification of a rare congenital anomaly of the myocardium by two-dimensional echocardiography: persistence of isolated myocardial sinusoids. Am J Cardiol. 1984, 53:1733-1734. 10.1016/0002-9149(84)90618-0

13. Paterick TE, Tercius AJ, Agarwal A, Treiber SC, Khandheria BK, Tajik AJ: Double jeopardy in the echocardiography laboratory: coexistence of two distinct cardiomyopathies?. Echocardiography. 2014, 31:931-935. 10.1111/echo.12502

14. Oechslin EN, Attenhofer Jost CH, Rojas JR, Kaufmann PA, Jenni R: Long-term follow-up of 34 adults with isolated left ventricular noncompaction: a distinct cardiomyopathy with poor prognosis. J Am Coll Cardiol. 2000, 36:493-500. 10.1016/s0735-1097(00)00755-5

15. Jenni R, Oechslin E, Schneider J, Attenhofer Jost CH, Kaufmann PA: Echocardiographic and pathoanatomical characteristics of isolated left ventricular non-compaction: a step towards classification as a distinct cardiomyopathy. Heart. 2001, 86:666-671. 10.1136/heart.86.6.666

16. Petersen SE, Selvanayagam JB, Wiesmann F, et al.: Left ventricular non-compaction: insights from cardiovascular magnetic resonance imaging. J Am Coll Cardiol. 2005, 46:101-105. 10.1016/j.jacc.2005.03.045

17. Jacquier A, Thuny F, Jop B, et al.: Measurement of trabeculated left ventricular mass using cardiac magnetic resonance imaging in the diagnosis of left ventricular non-compaction. Eur Heart J. 2010, 31:1098-1104. 10.1093/eurheartj/ehp595

18. Phan HT: Non-compaction cardiomyopathy misdiagnosed as dilated cardiomyopathy. JCR. 2018, 8:10-13. $10.17659 / 01.2018 .0004$ 